\section{Limitations of Rib Fixation for Chest Wall Reconstruction}

\section{Abstract}

Surgical rib fixation in the setting of flail chest has proven benefits, while surgical fixation of non-flail fractures is controversial. Chest wall reconstruction with rib fixation techniques can improve pain, preserve breathing mechanics and prevent complications from lung herniation. However, there are limitations to this technique. With this case report, we describe plate fracture and screw migration after attempting to reconstruct a chronic chest wall defect.

Keywords: Rib fixation; Multiple rib fracture

Received: February 24, 2016; Accepted: March 17, 2016; Published: March 25, 2016

\section{Introduction}

Though rib fixation in the setting of flail chest is widely accepted [1] rib fixation has expanding indications in non-flail rib fractures to improve pain, lung function, cosmetic result and chest wall deformity [2]. Titanium rib fixation devices have also been successfully used to treat chest wall defects [3]. We present an unfortunate case of titanium plate fracture and screw migration in the setting of rib fixation as well as a discussion on the limits of the technique.

\section{Case Presentation}

A 55 year old female with a history of smoking, COPD, chronic cough, obesity, and diabetes presented with four months of progressive right chest pain and dyspnea. A chest CT revealed isolated fractures of ribs $6,7,8$ and 9 on her right side with distraction of rib ends (Figure 1).The patient denied any recent trauma. She received oral narcotic and epidural delivery of pain medication with no improvement in intractable pain.

The patient underwent open reduction and rib fixation of right ribs $6,7,8$ and 9 with anterior titanium plate fixed with multiple bicortical screws on each rib (SternaLock ${ }^{\circledR}$ Blu Primary Closure System; BioMet, Warsaw, IN) resulting in marked improvement of her pain and dyspnea. However, ten days following surgery, the patient reported an abrupt, severe pain in the right chest with coughing. Chest X-ray showed right lower lobe pneumonia, fracture of the plate on the 7th rib, migration of several screws and displacement of plates on ribs 8 and 9 (Figure 2).

The patient was treated with antibiotics and removal of the damaged and migrated plates and screws. All soft tissue and ribs all appeared healthy, without signs of infection or necrosis.

\section{Cherie P Erkmen ${ }^{1}$ and Mengyi Zha}

1 Department of Thoracic Medicine and Surgery, Temple University Health System, 3401 N. Broad Street, Parkinson Pavilion, C-100, Philadelphia, PA, USA

2 Geisel School of Medicine, Dartmouth College, Hanover, NH 03755, USA

\section{Corresponding author: Cherie P Erkmen}

\section{Cherie.P.Erkmen@tuhs.temple.edu}

M.D., Associate Professor of Surgery, Temple University Health System, Department of Thoracic Medicine and Surgery, Parkinson Pavilion, Suite C-100, Philadelphia, PA 19140, USA.

Tel: (215) 707-9115

Fax: (215) 707-9131

Citation: Erkmen CP, Zha M. Limitations of Rib Fixation for Chest Wall Reconstruction. Insights Chest Dis. 2016, 1:2.
The patient recovered with baseline pain controlled with chronic narcotic medication.

\section{Case Discussion}

Rib fixation has been shown to decrease pneumonia, time in intensive care units, ventilator support, chest wall deformity, need for tracheostomy and medical costs, especially in the setting of flail chest [1]. A recent review of 9 studies supported the use of surgical stabilization in the management of multiple, non-flail and painful rib fractures, citing improved pain control, respiratory function, and quality of life with reduced socioprofessional disabilities [2]. Rib fixation for intractable pain, chest wall deformity, rib fracture non-union, and in the setting of thoracotomy for other indications has been reported, but remains controversial [3-5].

Chest wall defects pose a challenge in thoracic surgery. Repair requires addressing chronic pain, paradoxical breathing motion, restoring cosmetic contours and lung herniation [6]. Rigid prosthetic reconstruction can result in a restrictive physiology, while flexible prosthetic reconstruction does not adequately recreate the structure needed for breathing mechanics [3]. Reconstruction of the chest wall is a growing indication for rib 


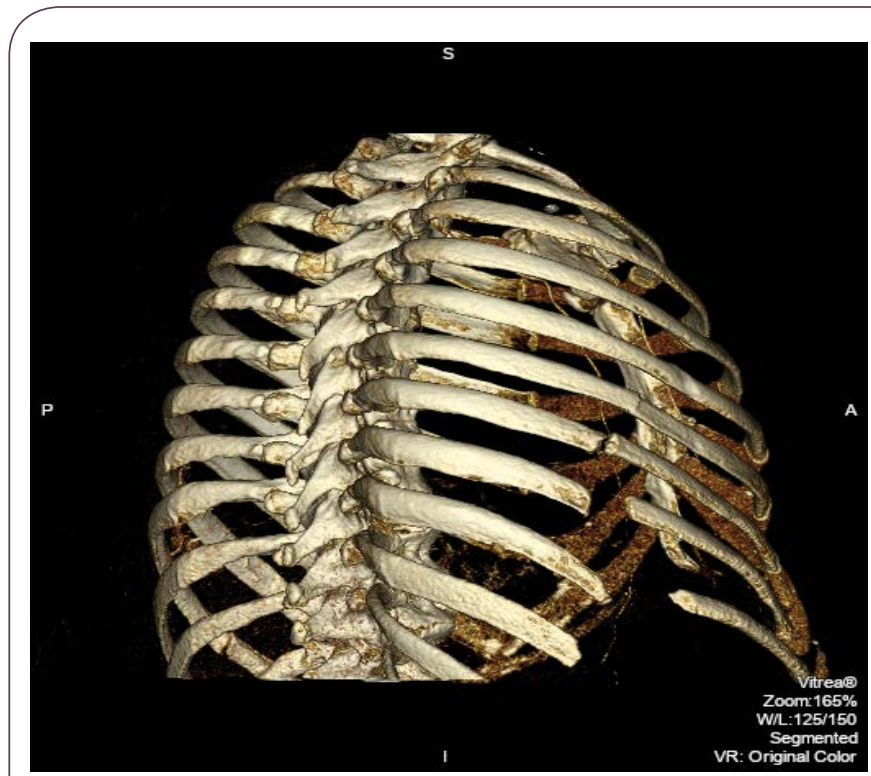

Figure 1 Vitrea ${ }^{\circledR}$ three dimensional reconstruction image of the postero-lateral view showing fractures of the right ribs 6 through 9 before rib fixation.

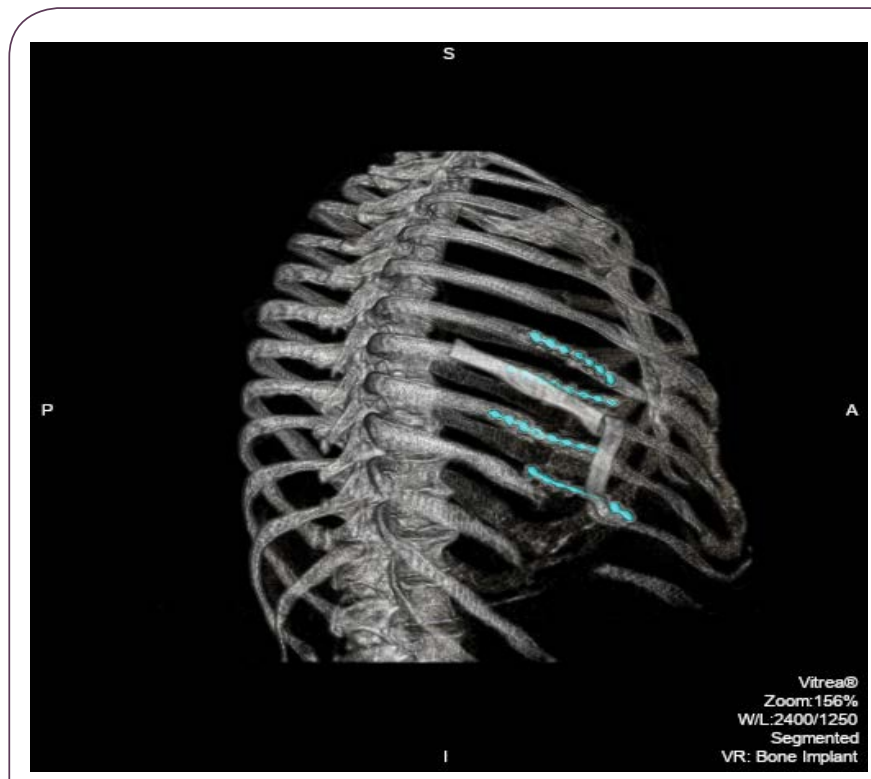

Figure 2 Vitrea ${ }^{\circledR}$ three dimensional reconstruction image of the postero-lateral view showing fracture of the plate on the 7th rib, migration of several screws and displacement of plates on ribs 8 and 9 (green arrows).

fixation techniques. Though conventional orthopedic practice advises against attempting to span large defects with rib fixation, there are reported successes of chest wall reconstruction using rib fixation [7].

With this case study, we hope to add to the growing experience of rib fixation for chest wall reconstruction by highlighting the limitations. We experienced plate fracture and screw migration and the circumstances in which this occurred. There are several contributing factors to the failure of this repair. First, the chest wall defect in our patient was large, spanning up to $5 \mathrm{~cm}$ at the $9^{\text {th }}$ rib. The interval time between the fracture and the rib fixation was likely several months based on evidence of chest wall remodeling and the obligate gap between the fractured ends. Though reconstruction of the ribs was possible with titanium plates and bicortical screws, the chronically remodeled tissues placed enough strain on the repair to cause fracture of a plate and migration of the screws. Alternative options for repair include wire sutures, intramedullary wires or plates, plates of various metal or absorbable materials and plates. A recent study demonstrated success in bridging chest wall defects with titanium clips in conjunction with a novel acellular collagen matrix (Veritas patch) [3]. However, the authors experienced failure when significant chest wall defects conferred tension on the repair. There is no established size guideline of chest wall defect that is safe to bridge. It is up to the surgeon to judge the amount of tension on a rib fixation based on the size and the surrounding tissues.

Second, our primary goal for rib fixation was to address chronic pain and relieve our patient from chronic narcotic use. It is our experience that the longer a patient endures pain for a rib fracture, the less likely we are to successfully treat that pain with surgery. Third, rib fixation is likely to fail in the setting of pneumonia which increases stress on the fixation through coughing. Traditionally, to view radiography will sufficiently diagnose pneumonia and plate migration or failure [8]. However, in the chest, CT scan may be better at demonstrating nonunion [9] and the possible local environment of pneumonia, abscess or empyema which may also require operative management. Lastly, patient factors including smoking, diabetes and chronic cough with COPD are all contributors to wound healing and failed fixation.

We believe that successful operative management of chest wall defects with rib fixation involves understanding the potential successes and failures. Rib fixation in the setting of a large chest wall defect, a long interval of time from the initial fracture, and for intractable pain diminishes the benefit. It is the responsibility of the surgeon to clearly define the chances of success based on patient factors in addition to technical context.

\section{Acknowledgement}

We are grateful to Julianna M Czum, M.D., for the threedimensional reproductions of the radiological films.

\section{Declaration}

The corresponding author accepts responsibility for the integrity of the submitted work and attests that no undisclosed authors contributed to the manuscript. 


\section{References}

1 Bulger EM, Arneson MA, Mock CN, Jurkovich GJ (2000) Rib fractures in the elderly. J Trauma. 48: 1040-1047.

2 Girsowicz E, Falcoz PE, Santelmo N, Massard G. (2012) Does surgical stabilization improve outcomes in patients with isolated multiple distracted and painful non-flail rib fractures? Interact Cardiovasc Thorac Surg 14: 312-315.

3 Billè A, Okiror L, Karenovics W, Routledge T (2012) Experience with titanium devices for rib fixation and coverage of chest wall defects. Interact Cardiovasc Thorac Surg 15: 588-595.

4 Nirula R, Diaz JJ Jr, Trunkey DD, Mayberry JC (2009) Rib fracture repair: indications, technical issues, and future directions. World $J$ Surg 33: 14-22.
5 Nirula R, Mayberry JC (2010) Rib fracture fixation: controversies and technical challenges. Am Surg 76: 793-802.

6 Weyant MJ, Bains MS, Venkatraman E, Downey RJ, Park BJ, et.al (2006) Results of chest wall resection and reconstruction with and without rigid prosthesis. Ann Thorac Surg 81: 279-285.

7 Westphal FL, Lima LC, Lima Netto JC, Seelig Sda C, Lima KF (2014) Chest wall reconstruction with titanium plates after desmoid tumor resection. J Bras Pneumol 40: 200-202.

8 Slone RM, MacMillan M, Montgomery WJ (1993) Spinal fixation-3: Complications of spinal instrumentation. RadioGraphics 13: 797-816.

9 Busam ML, Esther RJ, Obremskey WT (2006) Hardware removal: indications and expectations. J Am Acad Orthop Surg 14: 113-120. 